\title{
Keratoameloblastoma of Oral Cavity: Report of Two Cases
}

\author{
Manoj Prabhakar ${ }^{1}$, Sivapathasundharam B. ${ }^{2}$, Logeswari J. ${ }^{3}$, Manikandhan R. ${ }^{4}$ \\ ${ }^{1}$ Assistant Professor, ${ }^{2}$ Professor and Head, ${ }^{3}$ Associate Professor, Department of Oral Pathology, Meenakshi \\ Academy of Higher Education and Research, Faculty of Dentistry, Meenakshi Ammal Dental College, \\ Chennai-600095, ${ }^{4}$ Professor, Department of Oral and Maxillofacial Surgery, Meenakshi Academy of Higher \\ Education and Research, Faculty of Dentistry, Meenakshi Ammal Dental College, Chennai-600095
}

\begin{abstract}
Keratoameloblastoma is a rare variant and histologically heterogenous group of ameloblastomas, which have areas of ameloblastic epithelium and extensive keratin formation. Other subtypes of ameloblastoma which exhibit keratinisation in their parenchyma include acanthomatousameloblastoma, and papilliferouskeratoameloblastoma. Previously it was thought to be a variant of acanthomatousameloblastoma with focal areas of keratinisation. Extensive keratin deposition in the connective tissue isolates keratoameloblastoma as a separate entity from acanthomatousameloblastoma. The tumour presents itself as a destructive and an enlarging mass, mostly involving the posterior region of the mandible, with men being more commonly affected in the ratio of 3:1. This case report presents two cases of keratoameloblastoma, one in the posterior mandible and the other in the anterior maxillary region.
\end{abstract}

Keywords: Keratoameloblastoma, maxilla, mandible, oral cavity.

\section{Introduction}

Odontogenic tumours are the most common tumours of the head and neck region that occupy around 3\%$9 \%$ of all biopsied specimens, and may arise from or associated with odontogenic apparatus, their derivatives or their remnants. $\left[^{1}\right]$ Among the odontogenic tumours, ameloblastoma is considered to be the most common lesion next toodontoma in Asian population. $\left[{ }^{2}\right]$

Ameloblastoma is a true neoplasm of enamel organ type tissue, which is cytologicallybenign but clinicallyaggressive and destructive in its course. It is the most common odontogenic tumor of the jaw bones with various histologic sub-types; follicular and plexiform types being the basic histologic presentation.

\section{Corresponding Author:}

\section{Dr. Manoj Prabhakar}

Assistant Professor, Department of Oral Pathology, Meenakshi Academy of Higher Education and Research, Faculty of Dentistry, Meenakshi Ammal Dental College, Chennai-600095

e-mail: drmanoj.oralpathology@madch.edu.in
Other histomorphic variations include acanthomatous, granular, basal cell, desmoplastic and hemangiomatous. Except the last two, the histological variation occurs in the epithelial component. Desmoplastic ameloblastoma does not exhibit the usual peripheral palisading and central stellate reticulum type cells in the epithelial islands. In contrast the epithelial islands appear compressed with the connective tissue stroma made up of hypocellular dense collagenous fibrous tissue with hyalinisation. Further, the distinct site of occurrence and radiographic appearance of this variant made it to be considered as a separate clinical type in World Health Organization (WHO) classification for odontogenic Tumour, in 2005, however it has been re-included as a histological subtype in 2017 classification. $\left[{ }^{3}\right]$ In hemagiomatoustype the alteration is present only in the stroma by the presence of large, endothelial lined blood filled spaces.Amidst these variations, few of the subtypes exhibit keratinisation in their parenchyma, which include acanthomatousamelo blastoma, keratoameloblastoma and papilliferous keratoameloblastoma. $\left[{ }^{2,4}\right]$

Keratoameloblastoma is a term, which is used to describe a histologically heterogenous group of ameloblastomas, which have areas of ameloblastic 
epithelium and extensive keratin formation.[4] Pindborg in 1970 described the tumour islands with papilliferous projections and keratinizing cysts as papilliferouskeratoameloblastoma and the same type of tumour without papilliferous appearance was described by Altini as keratoameloblastoma. WHO in 1992, later considered this variant as acanthomatousameloblastoma with areas of keratinisation. $\left[{ }^{4,5}\right]$ Extensive keratin deposition in the connective tissue isolates keratoameloblastoma as a separate entity from acanthomatousameloblastoma. Both keratoameloblastoma and papilliferouskeratoameloblastoma are considered as extremely rare tumours with distinct histological features. $\left[{ }^{2}\right]$

Here we present two such cases of ameloblastoma with extensive keratinisation. Diagnosing these keratinising lesions and differentiating it from other keratin producing intraosseous neoplasms is a confrontable task and so are discussed in the light of this case report.

Case 1: A 74-year-old female patient reported with the complaint of swelling on her right lower jaw region for past 3 months; which was slow growing and was increasing in its size gradually over the period. The medical and familial history of the patient was noncontributory and there were no associated deleterious habits. Clinical examination revealed gross facial asymmetry with the swelling on right side of the body of mandible extending from the commissure of lip till the angle region. [Fig.1] The surface of the swelling appeared smooth with no evidence of sinus or discharge extra-orally. The lymph nodes were not palpable and the swelling was not associated with pain or paresthesia. Intra orally, the lesion was smooth, extending from the edentulous area of 45 to 48 region, with no surface ulceration. Orthopantamograph revealed a multilocular radiolucent lesion occupying the right body of the mandible, extending from 45 to 48 region. CBCT showed bi-cortical expansion of the right side mandible and erosion on the lingual and inferior surfaces. [Fig.2A, $\mathrm{B} \& \mathrm{C}]$ Fine needle aspiration was performed and no cystic fluid obtained from the swelling. Based on the history, clinical and radiographical features, a provisional diagnosis of benign odontogenic tumour was made with the differential diagnosis of ossifying fibroma. Incisional biopsy was performed and the specimen was sent for histopathological examination.

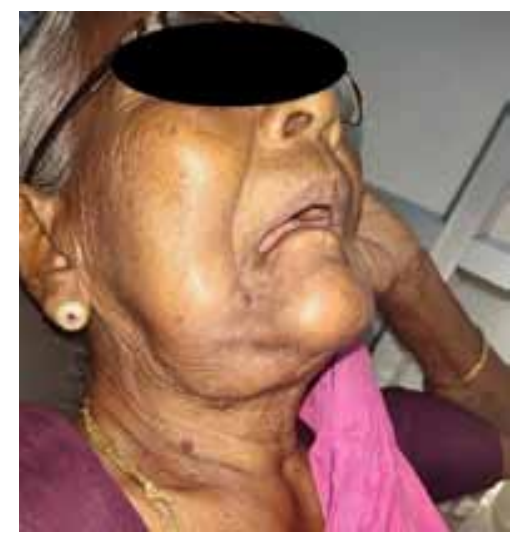

Fig.1: Swelling on right side of the body of mandible

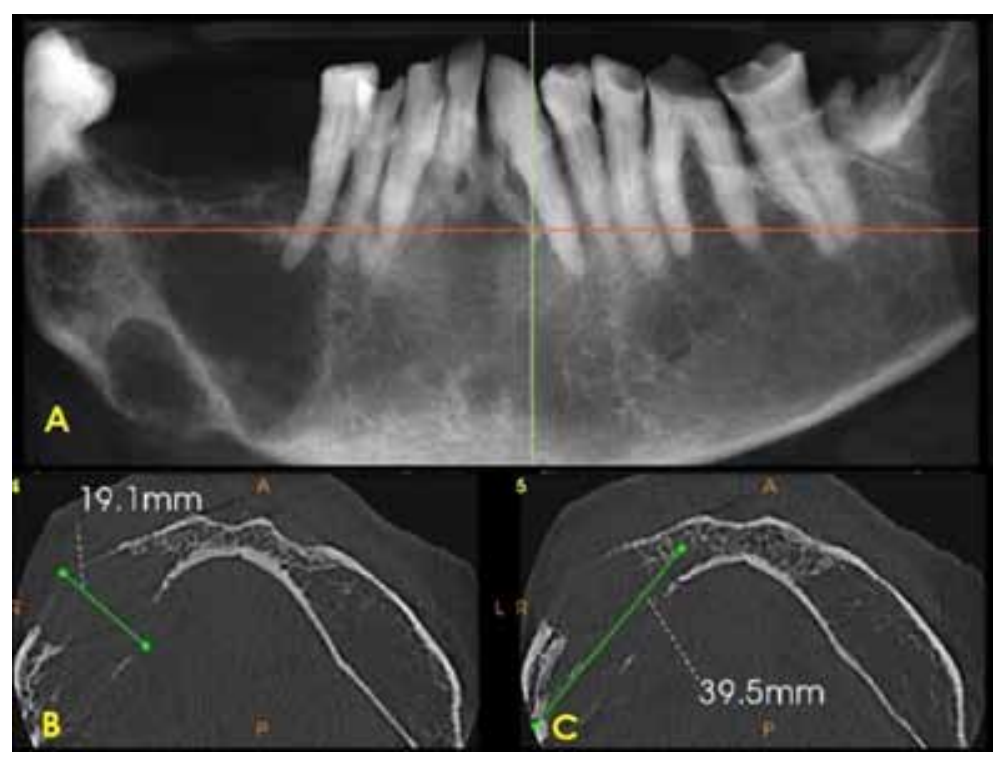

Fig.2A: OPG revealing multilocular radiolucent lesion occupying the right body of the mandible. 2B \& 2C: CBCT showing bi-cortical expansion of the right side mandible 
Grossing showed three greyish white soft tissue specimen, which were firm in consistency. Histopathological examination of the H\&E stained sections revealed an encapsulated lesion with dense connective tissue stroma. Numerous proliferating odontogenic islands in the form of follicular and plexiform pattern were noted. Most of these epithelial islands show extensive keratin occupying the entire follicle. Few islands showed straggling extension into the connective tissue giving a stretched-out appearance of the follicles at the extremities. [Fig.3A]

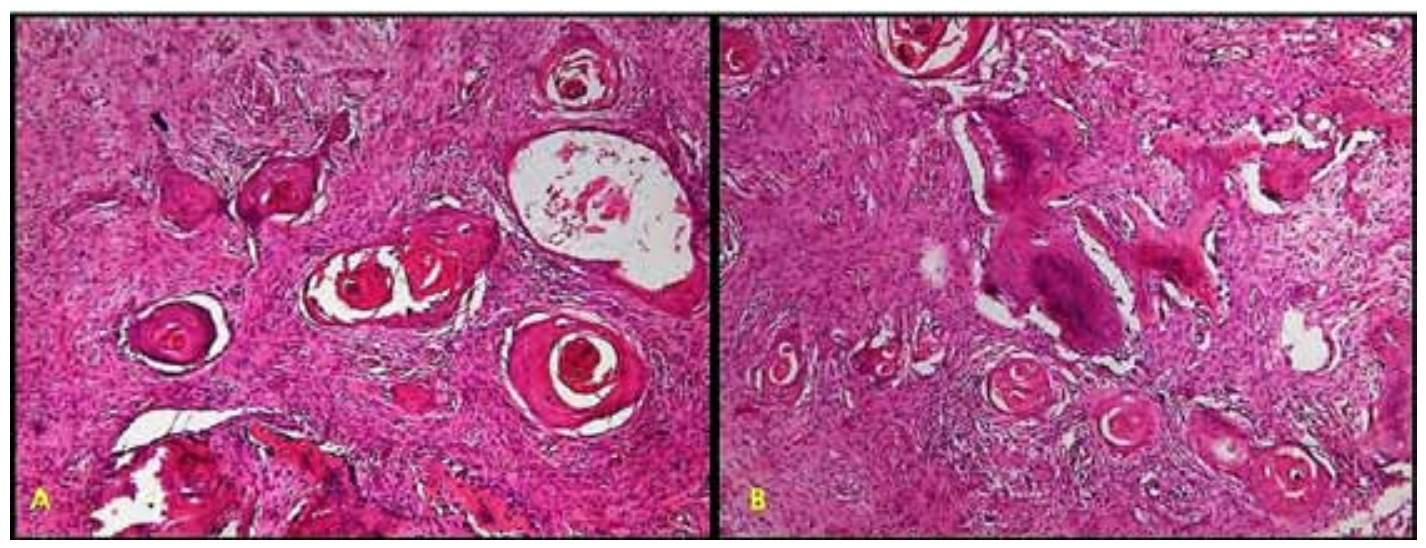

Fig. 3A: Keratin filled follicles with stretched-out appearance at the extremities. 3B: Areas of metaplastic bone formation representing osteoplasia

There were also areas of keratin in the connective tissue extruded from the epithelial islands. These keratin were arranged in a lamellar pattern, giving a 'pacinianlike stack of lamellatedparakeratin' appearance. Few areas of keratin filled cystic spaces were also evident. The connective tissue was fibrous with a very minimal inflammatory component. Areas of metaplastic bone formation were also evident representing osteoplasia. [Fig.3B] Based on these histologic features, a final diagnosis of keratoameloblastoma was made.
Segmental resection of right side of the body mandible was performed and the entire tumour was removed in toto.A bone clearance of $8 \mathrm{~mm}$ was given both the sides to prevent recurrence. Only a reconstruction titanium plate $(2.5 \mathrm{~mm})$ was placed as a reconstructive option as the patient had medical co-morbidities as contraindication for major bone flap reconstruction. A six month review showed no relapse and patient was kept on review for future reconstruction. [Fig.4A\&B]

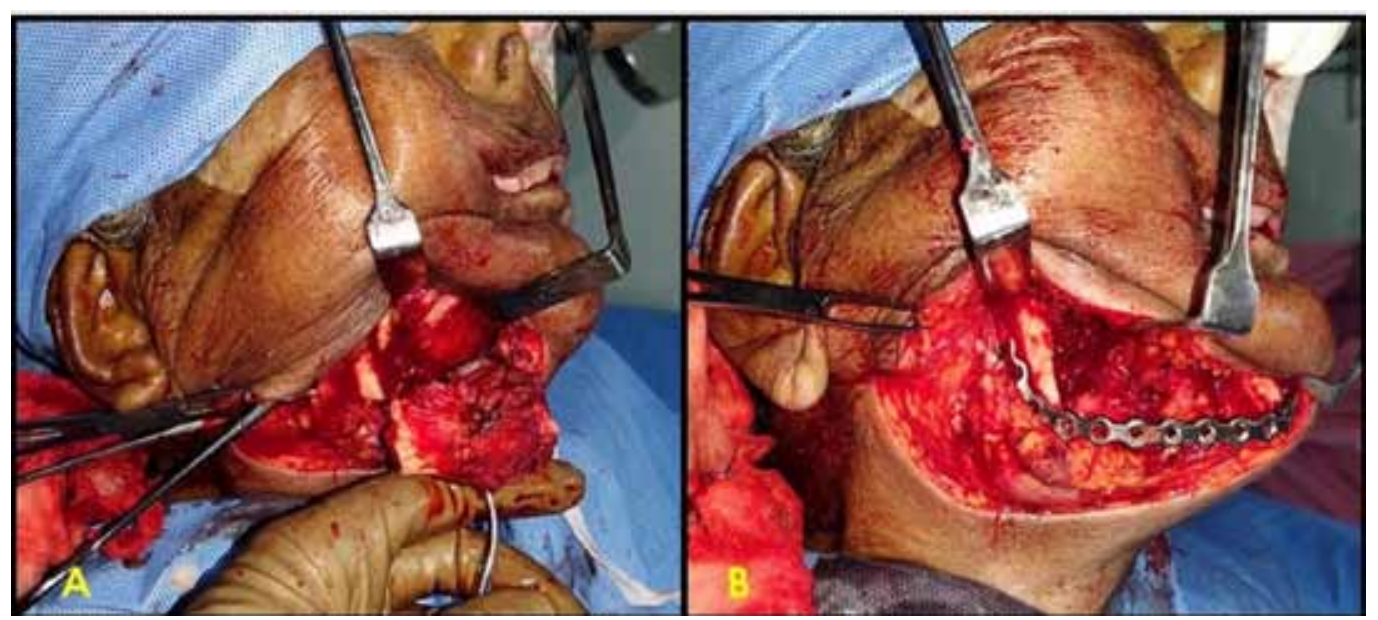

Fig.4A: Segmental resection of right side of the body mandible. 4B: Placement of reconstruction titanium plate 
The excised specimen had a gritty texture when cut during grossing. Histological features of the excised specimen were in confirmatory with the previous diagnosis of keratomeloblastoma.

Case 2: A 42-year-old male patient reported with a complaint of slowly expanding painless swelling on the right upper front tooth region. The size of swelling increased gradually to attain the size of $4 \times 4 \mathrm{~cm}^{2}$ within three months. Intra orally the swelling has expansion more towards the buccal side in relation to 11 to 15 region, extending up to the buccal vestibule, whereas the palatal expansion was very minimal. The swelling was hard on palpation with ill-defined margins. Radiograph revealed mixed radioopacities and radiolucencies with loculations. Together with clinical and radiological features, a provisional diagnosis of benign odontogenic tumour was made. Incisional biopsy was performed and the specimen was sent for histopathological examination.

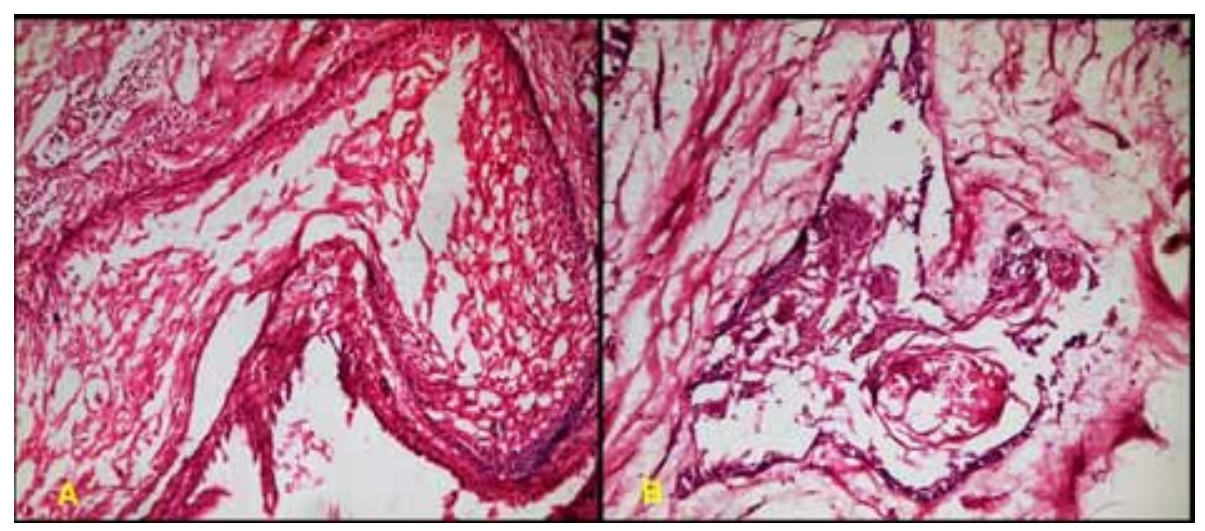

Fig.5A Ameloblastic island containing central stellate reticulum like cells 5B: Squamous cells replacing the central stellate reticulum like cells

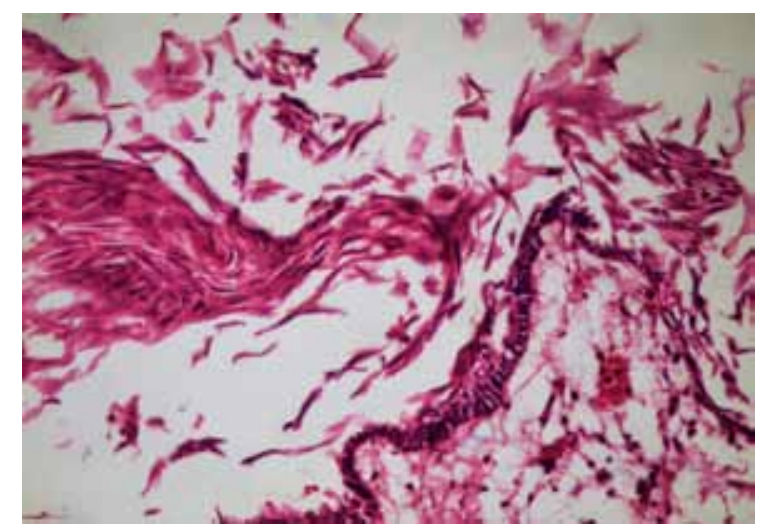

Fig.6: Extrusion of keratin outside the island, resembling shredded carrots

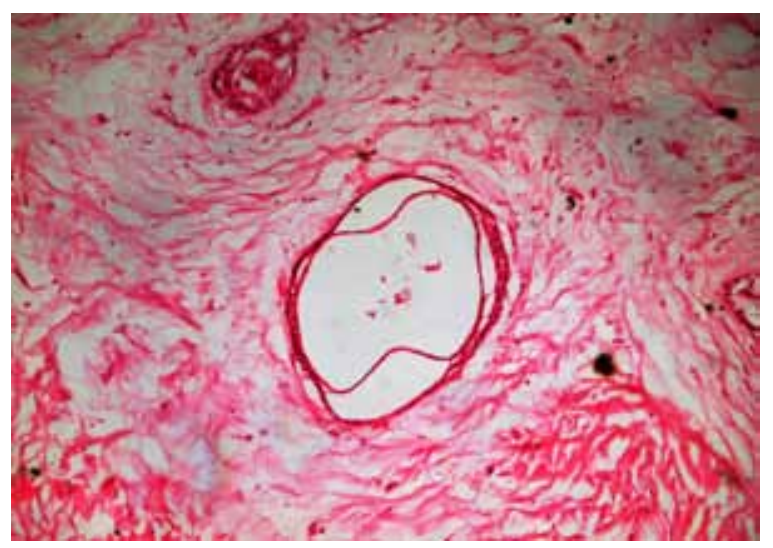

Fig.7: 'Pacinian- like stack of lamellatedparakeratin' appearance 
Microscopic examination of $\mathrm{H}$ and $\mathrm{E}$ stained sections revealed strands of odontogenic epithelium interspersed in the connective tissue stroma in the form of small islands and cords. The peripheral cells of the epithelial islands are made up of hyperchromatic, palisading columnar cells with reversal of nuclear polarity. Stellate reticulum like cells are present in the center of these islands. [Fig.5A]In few islands, the central stellate reticulum like cells are replaced by squamous cells. [Fig.5B]The connective tissue stroma was moderately vascular with thick irregularly arranged collagen fibres exhibiting desmoplasia. Further focal areas showed multiple large and small cystic spaces, lined by low columnar to flat cells, with central squamoid island containing esoinophillic, acellular discrete strands/bands of keratin. [Fig.6] Extrusion of keratin outside the island, resembling shredded carrots or lamellar pattern, giving a 'pacinian- like stack of lamellatedparakeratin' appearance was striking. [Fig.7] All the above histological findings suggested the diagnosis of keratoameloblastoma. Radical excision of the tumour was done and the histopathological examination of the excised specimen revealed features similar to the preoperative specimen and confirmatory to that of the preoperative histological diagnosis.

\section{Discussion}

Ameloblastoma is a highly polymorphic benign neoplasm of odontogenic origin, with its few variant undergoing different forms of metaplasia. This kind of metaplasticchangecanbeattributed tothemultipotentiality of the odontogenic epithelium. ${ }^{[5,6]}$ Robinson defined ameloblastoma as unicentric, nonfunctional, intermittent in growth, anatomically benign and clinically persistent. $\left[{ }^{2}\right]$ Its benign nature, grotesque size, locally invasive behaviour, and common occurrence make the lesion distinct from other odontogenic tumours.

Pindborg in 1970 first proposed the term Keratoameloblastoma (KA) and later WHO (1992) defined KA as an ameloblastoma with extensive keratinisation. However, WHO in 2005 classification for odontogenic tumours has not mentioned this term but considered this lesion as a variant ofacanthomatousameloblastoma with focal keratinisation. $\left[^{7}\right]$ The reason for keratoameloblastoma, failed to get placed in the WHO classification of odontogenic tumors, could be due to the lack of adequate number of case reports and no different biological behaviour from conventional ameloblastomas. Pindborg also noted few cystic areas with some papilliferous appearance of odontogenic islands and named it as papilliferouskeratoameloblastoma (PKA) $\left[{ }^{8}\right] \quad$ Some authors consider PKA as a sub-type of KA; however, both are considered as rare variants of ameloblastoma. $\left[{ }^{9}\right]$

There are about 19 cases of KA, which have been reported till date in the English literature. In general, KA, shows similar demographic distribution like conventional ameloblastoma in terms of age, sex and site. The age group ranges from 26 to 76 years with 44 years as a mean age, with men being more commonly affected in the ratio of $3: 1 \cdot\left[{ }^{5,10}\right]$

The tumour presents itself as a destructive and an enlarging mass, mostly involving the posterior region of the mandible including body and ramus, followed by posterior maxilla, anterior mandible and maxilla being the least. $\left[{ }^{10}\right]$ All the cases reported in the literature are intra-osseous with a very few report on its peripheral variant. Radiographically, it presents as a radiolucent lesion with marginal erosion and perforation of the cortical plates as is seen in the present case.

The varied histomorphology of KA, makes the lesion unique and enabled to categorise it histologically into four subtypes by Whitt,namely $\left[{ }^{9}\right]$ :

(i) Papilliferous histology: odontogenic epithelium shows papillary projections into the cystic spaces

(ii) Simple histology: odontogenic follicles are filled with parakeratin or ortho-keratin and lined by ameloblast-like cells showing reversal of polarity

(iii) Simple histology with KCOT(Kerato Cystic Odontogenic Tumour)-like features: shows similar features of simple type, in addition it contains features of conventional KCOT (however, in 2017 WHO Classification it was reverted back to cyst category). $\left[{ }^{11}\right]$

(iv) Complex histology: consists of epithelial follicles packed with parakeratin or orthokeratin, extrusion of keratin masses into connective tissue stroma in the form of "Pacinian-like stacks" with or without foreign body reaction.

Histopathological features in both the cases reveal a complex histology as there were numerous odontogenic epithelial islands arranged in the form of follicles, lined by hyperchromatic, palisading columnar cells with reversal of nuclear polarity, with the presence of 
stellate reticulum like cells in the center of these islands. Extensive keratin formation were noticed in most of the islands. The stroma appears highly collagenous, exhibiting marked desmoplasia. Areas of extruded keratin into the connective tissue were also seen, giving a 'pacinian- like stack of lamellatedparakeratin' appearance. Focal areas of large and small cystic spaces lined by low columnar cells with multiple clusters of mucous cells were also noticed. Few of the islands were not characteristically lined by odontogenic epithelial cells showing reversal of polarity validating the fact that few cases reported in the literature lack convincing histological evidence of typical ameloblastoma. $\left[{ }^{12,13}\right]$ Metaplastic bone formation, termed as osteoplasia, was also noticed within the collagenized fibrous connective tissue stroma.

A number of immunohistochemical (IHC) markers used for ameloblastoma, include CK 5/6, CK 13, CK 14 and CK 19. Each of these markers' expression vary according to stages of tooth germ development and subtypes of the tumour. Usually, CK13 is expressed in the stellate reticulum- like cells; CK 14 in the peripheral cells and CK 19 in all the cells of solid/multicystic and unicysticameloblastomas including acanthomatous and granular variant. This kind of expression does not hold the same for few variants like desmoplastic and peripheral ameloblastoma. Contrary to this view, the pattern of IHC expression was altered in the present case that the epithelial cells were not expressed by CK 14 and CK 19 markers. This negative expression suggests that origin of this tumour could even be in a very primitive stage of ameloblasts and need not be essentially in a later stage for its development. Molecular markers related to enamel proteins and transcription factors can be used to express these odontogenic epithelial cells in such scenario. $\left[{ }^{14}\right]$

Keratinisation in amelolastoma can be attributed to any of the following. It can evolve as a squamous metaplastic change in the central stellate reticulum-like cells in the ameloblastic follicles, as in acanthomatous variant; orcanarisedenovo as keratinising ameloblastoma; or due to ameloblastomatous transformation in the preexiting KCOT. $\left[{ }^{5}\right]$ The present case does not exhibit KCOT like areas, and so, the cause for keratinisation can be due to acanthomatousameloblastoma or de novo. However, straggling extension of the epithelial islands at the extremities, blending with the highly fibrous connective tissue, along with few areas of osteoplasia, triggers a thought of KA as a sequelae or the later stage of desmoplastic ameloblastoma or a hybrid variant.

Differentiating KA from other keratin producing intraosseous neoplasms (KPIONs) is a requisite at this juncture for a fitting diagnosis. The most debatable acanthomatousameloblastoma can be distinguished from KA by the presence of keratin within the follicles in acanthomatousameloblastoma, whereas, there will be extrusion of keratin into connective tissue stroma in KA. ${ }^{[15]}$ Absence of stromal keratinisation, classic tall columnar cells with reversal of nuclear polarityand stellate reticulum like cells draws out solid variant of keratocystic odontogenic tumor (SKCOT) from the spectrum of diagnosing KA. ${ }^{[16]}$ Primary intraosseous carcinoma (PIOC) is also a keratinising malignant neoplasm of jaw bones. Absence of connection with the oral mucosa and presence of varying dysplastic features distinguishes PIOC from KA. $\left[{ }^{5,6}\right]$

Treatment modality include wider excisionand segmental resection of the affected bone combined with post-treatment follow-up.Aggressive surgical resection along with $10 \mathrm{~mm}$ clearance may be necessary to prevent recurrence and reconstruction need diligent planning as the affected patients are usually of an older age. Recurrence is noted in a very minimal number of cases. The biological behaviour and prognosis of this lesion compared to other histological variants of ameloblastoma cannot be ascertained due to the limited number of cases and meagre follow-up. $\left[{ }^{5,10,17}\right]$

Keratoameloblastoma is a rare variant of conventional ameloblastoma and its biological behaviour is poorly understood. Having only a handful of cases in the literature, it requires strenuous learning for the pathologists to know its origin, course and recurrence. These kind of subtypes adds significance for academic interest but a knowledge of how these subtypes influence the nature of the tumor is a must requisite.

\section{Ethical Clearance: Nil}

Source of Funding: Meenakshi Academy of Higher Education and Research, Chennai, India

\section{Conflict of Interest: Nil}

\section{References}

1. Sivapathamsundaram B. Odontogenic tumor: Indian scenario. Journal of oral and maxillofacial pathology: JOMFP. 2017 Jan;21(1):2. 
2. Shafer H. Levy. Shafer's Textbook of Oral Pathology. B. Sivapathasundharam.

3. Sivapathasundharam B, Biswas PG, Preethi S. The World Health Organization classification of odontogenic and maxillofacial bone tumors: An appraisal. Journal of oral and maxillofacial pathology: JOMFP. 2019 May;23(2):178.

4. Parikh N, Nandini C, Jain S, Mansata AV. Peripheral keratoameloblastoma: A novel case report. Journal of oral and maxillofacial pathology: JOMFP. 2018 May;22(2):249.6

5. Bedi RS, Sah K, Singh A, Chandra S, Raj V. Keratoameloblastoma or Keratoodontoameloblastoma: report of its soft tissue recurrence with literature review. Quantitative imaging in medicine and surgery. 2015 Dec;5(6):898.

6. Kuberappa PH, Anuradha A, Kiresur MA, Bagalad BS. Papilliferous keratoameloblastoma-A rare entity: A case report with a review of literature. Journal of Oral and Maxillofacial Pathology: JOMFP. 2020 Feb;24(Suppl 1):S2.

7. Palaskar SJ, Pawar RB, Nagpal DD, Patil SS, Kathuriya PT. Keratoameloblastoma a rare entity: a case report. Journal of Clinical and Diagnostic Research: JCDR. 2015 Mar;9(3):ZD05.

8. Reichart PA, Philipsen HP. Odontogenic tumors and allied lesions. Quintessence Pub.; 2004 Jan.

9. Whitt JC, Dunlap CL, Sheets JL, Thompson ML. Keratoameloblastoma: a tumor sui generis or a chimera?. Oral Surgery, Oral Medicine, Oral
Pathology, Oral Radiology, and Endodontology. 2007 Sep 1;104(3):368-76.

10. Anajar S, Lakhbal A, Abada R, Mahtar M. Keratoameloblastoma of the mandible. European annals of otorhinolaryngology, head and neck diseases. 2017 May 1;134(3):205-6.

11. Speight P, Fantasia J, Neville B. Odontogenic and non-odontogenic developmental cysts. Dentigerous cyst. WHO Classification of Head and Neck Tumours. 4th Ed. Lyon: WHO. 2017:234.

12. Altini M, deV Slabbert H, Johnston T. Papilliferous keratoameloblastoma. Journal of oral pathology \& medicine. 1991 Jan;20(1):46-8.

13. Odell EW. Biopsy pathology of the oral tissues. Chapman \& Hall Medical. 1998;1998:453-63.

14. Hunter KD, Speight PM. The diagnostic usefulness of immunohistochemistry for odontogenic lesions. Head and neck pathology. 2014 Dec 1;8(4):392-9.

15. Adeyemi BF, Adisa AO, Fasola AO, Akang EE. Keratoameloblastoma of the mandible. Journal of Oral and Maxillofacial Pathology: JOMFP. 2010 Jul;14(2):77.

16. Ide F, Mishima K, Saito I. Solid-cystic tumor variant of odontogenic keratocyst: an aggressive but benign lesion simulating keratoameloblastoma. Virchows Archiv. 2003 May 1;442(5):501-3.

17. Sisto JM, Olsen GG. Keratoameloblastoma: complex histologic variant of ameloblastoma. Journal of oral and maxillofacial surgery. $2012 \mathrm{Apr}$ $1 ; 70(4): 860-4$. 